\title{
Multimodal Learning of Pheromone Locations
}

\author{
Meenakshi Pardasani, ${ }^{1}$ Shruti D. Marathe, ${ }^{1}$ Urvashi Dalvi ${ }^{1,2}$ and
}

Nixon M. Abraham ${ }^{1, *}$

1 Laboratory of Neural Circuits and Behaviour (LNCB), Indian Institute of Science Education and Research (IISER), Pune, Maharashtra, 411008, India.

2 Institute of Bioinformatics \& Biotechnology, Savitribai Phule Pune University, Pune, Maharashtra, 411007, India.

*Correspondence: nabraham@iiserpune.ac.in

Running title: Neural correlates of pheromonal memory

Number of Figures: 4

Number of Tables: 0

Keywords: Location preference, volatile pheromones, main and accessory olfactory bulb, non-pheromonal volatile discrimination 


\begin{abstract}
Memorizing pheromonal locations is critical for many mammalian species as it involves finding mates and avoiding competitors. In rodents, pheromonal sensing happens through both vomeronasal organ (VNO) and main olfactory epithelium (MOE). It remains unclear as to which modalities and cues are used by rodents to form these long-term memories efficiently. Here, we addressed this problem by training female mice on a multimodal task to locate pheromones by sampling volatiles emanating from male urine and associating with the dimensions of certain shapes sensed by their vibrissae. In this novel pheromone location assay, female mice' preference towards male urine scent decayed over time while permitting them to explore pheromones versus neutral stimuli, water. On training the animals for associations involving olfactory and whisker systems, they were able to memorize the location of opposite sex pheromones, when tested 15 days later. This memory was not formed either when the somatosensory inputs through whisker pad were blocked or pheromonal cues were replaced by that of same sex. On investigating the neural correlates of volatile pheromone information processing, we observed increased neurogenesis in the main olfactory bulb (MOB) after two weeks of learning. However, the pheromonal exposure induced Whitten effect, the estrous cycle synchronization, did not cause any differences in the MOB mediated discrimination learning pace for various non-pheromonal volatiles. Our study thus provides the evidence for associations formed between different sensory modalities facilitating the long-term memory formation in social and reproductive behaviors.
\end{abstract}




\section{Introduction}

In nature, when terrestrial rodents encounter pheromones sprayed on stones of different sizes and shapes, burrows and fallen leaves, how do they use different modalities to form a memory of the location of pheromones? Rodents are primarily macrosmatic mammals whose daily vital activities of foraging, finding mates and avoiding predators are dependent on olfaction (Doty, 2001). However, when it comes to memorizing scent marks, is their sense of smell enough or are associations being formed between multiple senses, integrating information to retain the memory of the marks? In rodents, coordinated action of sniffing and whisking has been observed during the exploratory behaviors (Moore et al., 2013; Welker, 1964). However, it has not been tested if such multi-sensory actions facilitate the long-term memory formation of pheromones. We decided to undertake an approach for probing the role of different systems governing this by using a newly designed 'multi-modal pheromonal learning' behavioral paradigm. Our paradigm lets the female mice use the sensory information processed by whisker pathway to associate a location presented with volatile and non-volatile pheromones over a location presented with a neutral stimulus (NS). Although the female mouse is allowed to have a direct exposure to soiled bedding, it can sample the volatiles emanating from male urine through a plate guarded by holes of a specific diameter. This paradigm offers us to probe for the role of whiskers and microvibrissae present on the snout along with the olfactory subsystems in forming the memory of pheromones location.

Classical knowledge reveals that detection happens via vomeronasal organ (VNO) and main olfactory epithelium (MOE) which further project to accessory olfactory bulb $(A O B)$ and main olfactory bulb (MOB), respectively. The output neurons project to bed nucleus of stria terminalis (BNST) and 'vomeronasal amygdala' and further to hypothalamic regions, which control the lordosis behavior in females (Halem et al., 1999; Isogai et al., 2011; Kimoto et al., 2005). Using genetic and lesion-based approaches, it has been found that $A O B$ senses non-volatile pheromones present in male urine and soiled bedding through its VNO receptors (Vomeronasal type 1, Vomeronasal type 2 and Formyl Peptide receptors) (Dulac and Torello, 2003) while the trace amine-associated receptors (TAARs) present on MOE projecting to MOB mitral cells detect air-borne, volatile pheromones (Liberles and Buck, 2006). Another receptor type includes MS4A receptors of "necklace" glomeruli subsystem found in caudal MOB, which are activated by certain pheromones such as 2,5dlmethylpyrazines (Greer et al., 2016). Such parallel mechanisms occurring via different olfactory subsystems reflect the complexity of pheromonal information processing by activating combination of receptor subtypes in rodents (Stowers and Logan, 2010).

Male urine scent-marking is an excellent example of intra and inter-sex social communication (Arakawa et al., 2008) leading to display of dominance by the giver (Humphries et al., 1999; Hurst, 1993) or attractive/preferential behaviors by recipient females (Roberts et al., 2014). In the context of mating, pheromones are important to be learnt and remembered by female mice. Earlier studies suggested that repeated vagino-cervical stimulation along with the pheromonal exposure is required for acquisition of long-term memory in females (Ichikawa, 2003). Although, now we know that a single exposure is enough to form a memory (Roberts et al., 2014), we 
do not yet understand the involvement of different sensory modalities in governing the memory formation/acquisition.

Under natural contexts, female mice will have to make active attempts in accessing the urine scent marks deposited by males. Thus, our paradigm involves probing the effect of pheromone priming on the chemo- as well as whisker-mediated investigatory behavior and long-term memory formation in female mice. We observed that the preference towards a zone containing pheromones decayed over time when mice were freely exploring the arena during the initial 4 days. Further, we trained female mice to learn the associations involving male pheromones, both volatiles and non-volatiles, and whisker-mediated sensory cues. These mice exhibited long-term memory when tested 15 days after the training, in the absence of any sensory cues. In order to investigate if somatosensation is important for locating such marks, we checked if the preference towards male mouse pheromones is negatively affected when the sensory input through whiskers and skin is blocked during the 15 days of conditioning. Interestingly, we saw poor pheromonal long-term memory in these females suggesting the involvement of somatosensory system along with MOE and VNO. Our data thus provide evidences for novel associations between different sensory modalities in facilitating long-term memory formation of pheromones.

\section{Methods}

Subjects: A total of 56 C57BL6/J female (10-14 weeks) and 4 C57BL6/J male mice (10-14 weeks) were utilized for the study. Three different groups of female mice were tested on 'multi-modal pheromonal learning' paradigm. Group 1 consisted of 8 whisker intact female mice trained for associating Male soiled Bedding and Urine volatiles (MBU) with a particular hole diameter punched on the plate guarding the chamber 1, where the stimulus is kept. Group 2 had 8 female mice, whose whiskers were trimmed during the training phase for the associations involving MBU. But with the same group, whiskers were kept intact during initial testing and memory testing phases. Group 3 consisted of 8 whisker intact female mice trained for associating Female soiled Bedding and Urine (FBU) with a particular hole diameter. All three groups were trained using the same paradigm and apparatus with same specifications. 6 animals from MBU and FBU whisker intact groups were used for adult neurogenesis quantification. 4 mice each from these groups were utilized for measuring sniffing behavior towards pheromonal volatiles. Male urine was collected from 2 male mice housed together (C57BL6/J, aged 10-12 weeks), who never had a sexual experience. Female urine was collected from 2 female mice housed together (C57BL6/J, aged 10-12 weeks), who were also never sexually experienced. Soiled bedding was collected freshly just before the experiments from their respective cages.

For non-pheromonal odor discrimination training, a total of $30 \mathrm{C57BL6/J}$ female mice, aged 8-14 weeks, were used. These were divided equally in two groups, one group (experimental) exposed to MBU every-day during the discrimination training period while the other group (control) unexposed. 12-hours light/dark cycle was maintained and mice were grouped in individually ventilated cages in a temperatureand humidity- controlled animal facility. All animal care and procedures were in accordance with the Institutional Animal Ethics Committee (IAEC) at IISER Pune and 
the Committee for the Purpose of Control and Supervision of Experiments on Animals (CPCSEA), Government of India.

\section{Multimodal Pheromonal learning:}

Apparatus: It consisted of an arena $(60 \mathrm{~cm} \times 30 \mathrm{~cm} \times 15 \mathrm{~cm}$, length $\times$ width $\times$ height, black colored and matt finished), which was divided into 3 equally spaced zones. Two $10 \mathrm{~cm} \times 10 \mathrm{~cm} \times 15 \mathrm{~cm}$ chambers guarded by removable plates were placed at the opposite ends of the length of arena. One removable plate guarding a particular chamber had equidistant $10 \mathrm{~mm}$ diameter holes on it, keeping other three sides of each chamber devoid of any holes. Chamber on the opposite side was guarded by a removable plate having equidistant $5 \mathrm{~mm}$ diameter holes. This allows the animal to sample the volatiles emanating from the chamber through only one (front) side. One of each kind guards these chambers when the animal was sampling the volatiles during the entire experimental duration (initial testing, training and memory testing). The apparatus was custom-made using black acrylic sheets $(6 \mathrm{~mm}$ thickness for the walls and $10 \mathrm{~mm}$ thickness for the base). Each chamber contained a $55 \mathrm{~mm}$ petri-dish filled with $100 \mu \mathrm{L}$ urine (attractive pheromonal stimulus in chamber 1) or water (neutral stimulus in chamber 2) (Figure 1B). Two different apparatuses were used for carrying out the multimodal pheromonal learning paradigm in the MBU and FBU groups.

Paradigm: The experimental design involves an initial testing phase of 4 days, training phase of 15 days and the testing of memory at $15^{\text {th }}$ day and $30^{\text {th }}$ day post training (Figure 1A). The initial testing phase was to investigate if female mice exhibit innate preference towards a zone presented with attractive volatile and non-volatile pheromones from male mice (Zone 1) over a zone containing water stimulus (Zone 2). To remove any directional bias towards a particular zone, the apparatus was rotated by $180^{\circ}$ everyday during the initial testing and training phases. Mice were equally distributed between the plate hole diameter and the volatile cue pairing (Urine $/ 5 \mathrm{~mm} \mathrm{v} / \mathrm{s}$ Water $/ 10 \mathrm{~mm}$ and Urine $/ 10 \mathrm{~mm} \mathrm{v} / \mathrm{s}$ Water $/ 5 \mathrm{~mm}$ ) to remove any possible bias towards specific hole diameter. The chambers remained the same during this counter-balancing, only new plates were used. Training was done following the initial testing phase for a period of 15 days. Each day, the animal was restricted in both the zones for a period of 15 minutes each (alternating between the two zones after every 5 minutes). To check for the multi-modal memory on $15^{\text {th }}$ and $30^{\text {th }}$ day, 'urine with soiled bedding' and water stimuli were removed from the chambers of the apparatus while keeping the plates of specific diameter holes undisturbed. The time spent in each of the zones, specifically in front of chamber 1 and chamber 2 were calculated using EthoVision software (Noldus Information Technology). The nose point feature (used to track animal) on EthoVision is used to visualize the tracks taken by an animal (Figure $1 \mathrm{D} 1$ and D2) and to calculate the time spent. Number of active attempts on the plates guarding chamber 1 and 2 were calculated manually by considering a nose poke into the hole as one attempt.

In case of testing the involvement of somatosensation in pheromone location learning and memory, an anesthetic gel (Lignocaine Hydrochloride gel- Lox-2\% jelly) was applied on the snout of the group 2 mice during the training phase of 15 days. Whisker trimming was done at a frequency of 5 days from the start of the training phase. Whiskers were allowed to grow and were never trimmed again after the training period. Mice had re-growing whiskers when the day $15^{\text {th }}$ memory was tested, 
thereby allowing us to specifically check for the role of somatosensation in mediating the association of pheromonal cue with hole-size.

\section{Go/no-go odor discrimination:}

Odors: Odors used were 1,4-Cineol (Cl), Eugenol (EU), Amyl acetate (AA), Ethyl butyrate (EB), Benzaldehyde (BZ), Nonanol (NN), Hexanal (HX) and 2-Pentanone (PN). The odors were diluted to $1 \%$ in mineral oil and further diluted 1:20 by airflow. All odors were bought from Sigma-Aldrich and mineral oil was bought from Oswal Pharmaceuticals, Pune, Maharashtra, India.

Apparatus: All experiments were done using two custom-made eight channel olfactometers controlled by software written in Igor Pro (Wavemetrics, OR). Briefly, mouse was put into an operant chamber, which had a combined odor sampling and reward delivery port on one end of the chamber. This ensured tight association of reward with the odor presented in a trial. Inside the odor port, a lick tube was placed on which the animal needs to lick to get the reward. Insertion of animal's head in the port resulted in breaking of an IR beam guarding odor/reward port, which initiated the trial. The odor and final (diversion) valves controlled the flow of odor in a timedependent manner, allowing opening of final valve $500 \mathrm{~ms}$ after odor valve opening. Each rewarded (S+) and non-rewarded (S-) odor was presented through either of the two valves. A total of four odor valves for two odors were used. The apparatus and the task design followed is same as done in previous published studies (Figure 4A1, A2, A3) (Abraham et al., 2010; Abraham et al., 2012; Abraham et al., 2004; Abraham et al., 2014)

Task habituation phase: First week post-water restriction involved training with the standard operant conditioning procedures. Initially, mice were rewarded with water upon insertion of head into the sampling port and breaking the IR beam. The difficulty levels increased gradually, wherein the animal needed to lick on the tube to get the reward. At the end of this phase, odor valve was introduced and the air passed through the mineral oil (solvent used for odorants) bottle was used as the stimulus.

Discrimination training phase: As the animal initiated the trial by breaking the beam, one of the odor valves and a diversion valve (DV) open for certain duration. The DV helps to divert the odorized air to the exhaust for $500 \mathrm{~ms}$ and thereafter to the odor port for the pre-decided stimulus duration $(2 \mathrm{~s}$ for the odor discrimination training). For a rewarded (S+) odor to be correctly registered, animal needs to lick any 3 out of 4 bins of $500 \mathrm{~ms}$ to get the reward. For an unrewarded odor (S-) to be correct, animal is allowed to lick at most 2 out of 4 bins. No punishment is given for a wrong response towards S- odor. There is a fixed inter-trial interval (ITI) of 5s during which the animal cannot initiate the next trial. Usually an optimally motivated animal took more than 10s as the ITI. Odors were presented in a pseudo-randomized manner (not more than 2 successive presentations of same odor and $\mathrm{S}+/ \mathrm{S}$ - odors were distributed equally in a block of 20 trials).

Resistance to memory extinction task: Once the animals reached a criterion performance of $>80 \%$ for an odor pair for which long-term memory needs to be checked, we performed resistance to memory extinction. This was done to stabilize 
the memory of an odor pair and is based on the 'Partial reinforcement theory' (Bouton and Sunsay, 2001). The task comprised of 100 trials with 50 trials for S+ and $\mathrm{S}$ - each. The $\mathrm{S}+$ trials are reinforced pseudo-randomly only for half of the trials as compared to a normal reinforcement learning where all $\mathrm{S}+$ trials are rewarded. Such a partial reinforcement learning increases the attention of the animal as the factor of anticipating a reward is no longer true for some trials and that the trial outcomes cannot be expected by the animal. This increases the association strength and helps preventing memory extinction.

Memory task: The olfactory memory was checked for AA v/s EB, 30 days after carrying the resistance to memory extinction task. The memory task comprised of 200 trials, first 60 trials of background odor pair and subsequent 140 trials with interleaved memory trials [4 trials (2S+ and 2S-) in every block of 20 trials of background odor pair]. The memory task was performed only after the accuracy of background odor pair reached $>80 \%$ for all animals. For checking AA/EB memory, BZ vs NN was used as a background odor. Memory trials were not rewarded. Memory is calculated as an average accuracy percent of 28 trials (14 S+ and $14 \mathrm{~S}$ - trials) tested over a total of 140 trials.

Sniffing behavior towards pheromones: To investigate the sniffing strategies of female mice towards male urine and female urine volatiles under whisker trimmed $\mathrm{v} / \mathrm{s}$ intact conditions, a head-restraining method was used to precisely deliver the volatiles on the snout of the mice (Bhattacharjee et al., 2019). These were subsets of mice utilized for multimodal pheromonal learning assay and their sniffing strategies were tested 2 months after their $30^{\text {th }}$ day memory was investigated. They were implanted with a head-post (custom-made head fixation set-up) on their head. The skin overlaying the skull was removed and head post was fixed using dental cement (Tetric N-Ceram, Ivoclar Vivadent clinical). Surrounding exposed skull was covered using the dental acrylic cement (DPI RR Cold cure, acrylic repair material). This surgery was performed under anesthesia maintained by intraperitoneal injection of Ketamine (Celtamin, Celtiss therapeuticals) 60mg/kg and Xylazine (Vea Impex) $10 \mathrm{mg} / \mathrm{kg}$. The eyes were protected with artificial tears to prevent dryness. Recovery time of two days was given and the weights were measured every day. Upon recovery, they were placed in a plastic tube and head-post was screwed onto a metallic device fitted on a platform. Urine volatiles and air presentations were done in a pseudorandom fashion through a nozzle. $100 \mu \mathrm{L}$ of urine was used for the same. Their sniffing behavior towards the volatiles was recorded using airflow pressure sensor (AWM2300V, Honeywell) (Bhattacharjee et al., 2019). Each presentation was carried out for a period of $2 \mathrm{~s}$ done over 10 such presentations of the same stimulus (total 20 presentations), accounting for a total of 20 s time of exposure to pheromonal volatiles on a single day.

\section{Adult neurogenesis quantification:}

Bromodeoxyuridine (BrdU) injection protocol: BrdU ( $\mathrm{TCl}$ chemicals, 98\% purity, dissolved in $0.9 \%$ Saline), a DNA synthesis marker (Gratzner, 1982) was injected intraperitoneally in mice at $100 \mathrm{mg} / \mathrm{kg}$ of body weight dosage four times in a day. These animals were perfused and brains were dissected out 4 weeks after they received injections. This ensures maximum number of BrdU labelled neurons to be traversed and integrated into the Olfactory Bulb $(\mathrm{OB})$ circuitry, thereby, making this a 
useful strategy to study the survival of adult-born neurons (Wojtowicz and Kee, 2006).

Immunohistochemistry: Mice received Thiopentone injections intra-peritonially (Thiosol sodium, Neon laboratories, $50 \mathrm{mg} / \mathrm{kg}$ ) to achieve deep anaesthesia state in order to carry out perfusion. Transcardial perfusion was done using 1X Phosphate buffered saline (PBS) followed by $4 \%(\mathrm{w} / \mathrm{v})$ Paraformaldehyde (PFA). Brain of the animal was dissected and incubated in $4 \%$ PFA overnight. Next day, tissue was washed twice with $1 \mathrm{X}$ PBS to remove extra fixative. In order to carry out cryotome sectioning, brain was cryopreserved using $20 \%$ sucrose $(\mathrm{w} / \mathrm{v})$ for a day and put on a shaker. It was then transferred to $30 \%$ sucrose $(\mathrm{w} / \mathrm{v})$ at $4^{0} \mathrm{C}$ overnight. Brain tissue was embedded in optimal cutting temperature (OCT) medium (Leica) and sectioned in a cryotome. $50 \mu \mathrm{m}$ coronal sections were cut. Freely floating sections were selected at every $300 \mu \mathrm{m}$ thickness from most rostral OB section being the first for immunostaining. Thus, a total of $9 / 10$ sections per $O B$ were utilized. Non-specific signal was blocked using PBS containing 5\% Normal goat serum (NGS) (Abcam, ab 7481) and $1 \%$ Triton-X (Sigma). Sections were further incubated with primary antibodies- Rat anti-BrdU (Biorad, MCA2060) and Chicken anti- NeuN (Millipore, $A B N 91)$ at 1:1000 dilutions. For BrdU staining, sections were pre-treated with $1 \mathrm{~N}$ $\mathrm{HCl}$ for 45 minutes at $37^{\circ} \mathrm{C}$ for mild DNA denaturation. Incubation was done at $4{ }^{0} \mathrm{C}$ for 22 hours. 1X PBS washes were given thrice (15 minutes each) after incubation period. Secondary antibodies used were Donkey anti-rat Alexa Fluor 488 (1:500 dilution), and donkey anti-chicken Alexa fluor 647 (1:500 dilution) (Jackson Immunoresearch, USA, code: 712-544-150 and 703-605-155, respectively). Sections were incubated in this cocktail of secondary antibodies for 2 hours at room temperature (RT). Sections were washed with 1X PBS and labelled with DAPI (Sigma, 1:500). They were mounted on glass slides using Vectashield medium (Vector labs).

Confocal Imaging and analysis: For quantification of BrdU labelled nuclei in MOB and $A O B$, imaging using SP8 confocal microscope (Leica) was done. Images were analyzed using Fiji ImageJ (Schindelin et al., 2012) using the 'analyze particles' plugin which upon thresholding on a binary image assigns individual cells with a number. The number was confirmed using its 'count cell' tool. The number of BrdUpositive cells was obtained for a total of $18 \mathrm{MOB}$ and 10-12 AOB sections from a single mouse ( $n=3$ mice for group 1 and 3 ). Imaging was done using $10 x$ objective lens at $0.75 \times$ zoom, at frame dimensions of $1.55 \mathrm{~mm} \times 1.55 \mathrm{~mm}$. Z-stacks were obtained at slice interval of $1 \mu \mathrm{m}$. Total number of BrdU-positive cells was then calculated as a function of $/-\mathrm{mm}^{3}$ [=(total number of cells)/(number of sections $\times 1.55$ $\times 1.55 \times 0.05)]$.

Statistical analyses: All data are represented as Mean \pm S.E.M (Standard Error Mean) and analysed using Graphpad Prism 8.0 (Graphpad Software Inc, USA). Normality of the data was checked using Shapiro-Wilk test. For data that followed normal distribution, Unpaired student's t-test, Paired student's t-test, One-way Analysis of Variance (ANOVA) and two-way ANOVA with post hoc Bonferroni's multiple comparison testing were carried out wherever required. All t-tests were twotailed. For data that did not follow normal distribution, Mann-Whitney and Wilcoxin matched pairs signed rank test were carried out wherever required. * represent $p<$ $0.05,{ }^{* *} p<0.01,{ }^{* * *} p<0.001$ and ${ }^{* * * *} p<0.0001$.

\section{Results and Discussion}


In their natural habitats, rodents may locate scent marks associating with different surrounding objects of various shapes and textures which raises the possibility for the stimulation of whiskers and microvibrissae during this process. Can then, the memory formation for location of scent mark be facilitated by the activation of whisker system along with the olfactory subsystems? In order to answer this, we designed a novel 'multimodal pheromonal learning' paradigm to test the preference of female mouse towards the urine of opposite sex (Figure 1A). It involved a set-up consisting of zone 1 and zone 2 . A $55 \mathrm{~mm}$ petri dish containing male urine was kept in the chamber 1 [used henceforth as opposite sex pheromones (OSP) chamber] of zone 1. Neutral stimulus (NS), water was kept in a petri dish in chamber 2 [used henceforth as NS chamber] present in zone 2. A mouse can sample the volatile stimuli through specific diameter holes made on one side of the chamber as depicted in figure 1B. To avoid any bias towards specific chamber, hole diameters were counterbalanced across mice for sensing male urine and water. Separate plates of a particular hole diameter were used in these conditions.

To investigate mice' (Group 1) innate preference towards any zones, they were allowed to explore both zones for a total of 10 minutes everyday during the initial 4 testing days. To remove any possible directional bias shown by the mice towards a particular zone, the apparatus was rotated by $180^{\circ}$ everyday during the initial testing and training days (See methods). The preference was quantified based on the time animal spent in front of OSP chamber while sensing the non-volatile pheromones from male soiled bedding (white line demarcated region in Figure 1B) and the volatiles emanating from the OSP chamber. When the female mice were sensing attractive pheromonal volatiles, we observed them making repetitive snout pokes into the holes of the plate guarding OSP chamber containing male urine. This experimental design facilitated the multi-modal learning of pheromone locations with the stimuli processing through the main olfactory and whisker systems. Thus, another parameter to measure the preference was the number of active attempts or snout pokes into the holes of specific diameter to sense the volatiles. Decayed preference was observed over the course of 4 consecutive initial testing days. On day 2 of the initial testing phase, we observed an increase in the time spent towards OSP chamber indicating the preference towards male urinary pheromones, which was reduced during the remaining two days (Figure 1C2: $p>0.05, F=4.34$ Repeated measures (RM) Two-way ANOVA). (Figure 1C2: RM Two-way ANOVA, Bonferroni's multiple comparison test, $p<0.05$ for day 2 and $p>0.05$ for all other days). Similarly, on initial days, i.e. day 1 and day 2, female mice made higher number of active attempts to sample the urinary volatiles. The number of attempts, however, became similar to the exploration behavior on water side as the days progressed (Figure 1C2: $\mathrm{p}>0.05, \mathrm{~F}=4.1$, RM Two-way ANOVA; Bonferroni's multiple comparison test, $p<0.01$ for day 1 and day 2, $p>0.9$ for day 3 and day 4). To test if this overall decline is due to variable responses shown towards both stimuli, we quantified and compared their attraction specifically towards the pheromones. Indeed, female mice spent lesser time (Figure S1A: $p<0.01, F=6.3$, RM One-way ANOVA; Bonferroni's multiple comparison test, $p<0.05$ for Day $2 \mathrm{v} / \mathrm{s}$ Day 3 and Day 2 v/s Day 4) and made fewer active attempts particularly towards OSP chamber as the days progressed during the initial testing phase (Figure S1B: $p$ $<0.0001, \mathrm{~F}=16.3$, RM One-way ANOVA; Bonferroni's multiple comparison test, $\mathrm{p}<$ 0.01 for Day $1 \mathrm{v} / \mathrm{s}$ Day 3, 4 and Day 2 v/s Day 3, 4). 
In order to investigate if the association of non-volatiles and volatiles through olfactory and whisker systems can be strengthened over time, we further trained mice for a period of 15 days after their initial testing period was over. Each mouse was trained for a total of 30 minutes in the set-up. During this time, a mouse is allowed to enter only one of the zones and the door is then closed for 5 minutes ensuring the intended associative learning of pheromone locations with the volatiles and the specific hole size. This is then alternated for both sides leading to a total of 15 minutes being spent on each side. Indeed, this restriction to one particular zone allowed mice enough time to explore the hole size, sense the volatiles and the nonvolatiles from bedding. To investigate if such a multi-modal association can be retained, we checked for pheromonal memory at Day 15 and Day 30 post training phase. Exposure to pheromonal stimuli was not allowed during the time period after the training. In case of female mice being trained with $\mathrm{MBU}$, associating male soiled bedding and urine v/s water, we observed an increased preference as measured by the two parameters: time spent and number of active attempts (Figure 1D1, D2). This confirms that mice had intact memory at Day 15 (Figure 1E1, E2: $p<0.01$ for time spent, Wilcoxin matched pairs signed rank test and $p<0.001$, Paired two-tailed

A

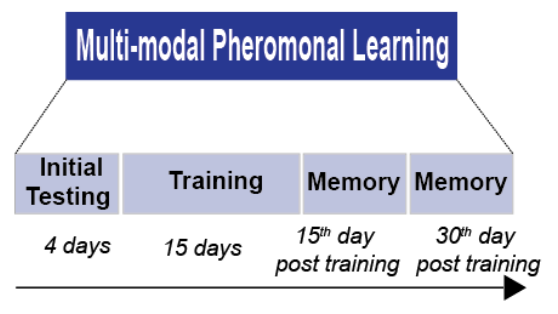

C1

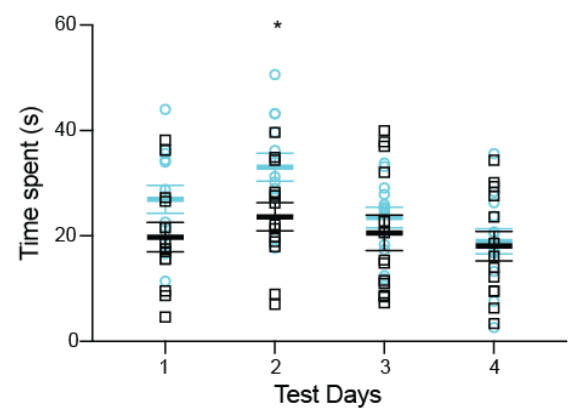

D1

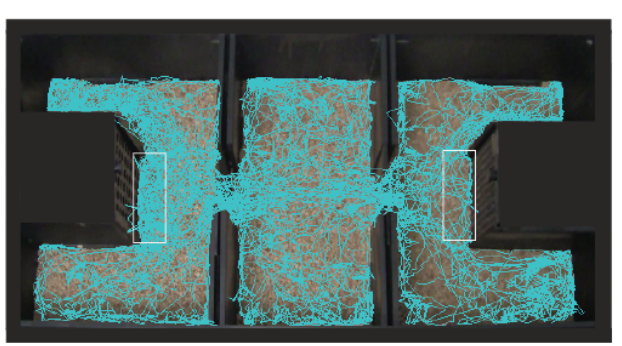

E1

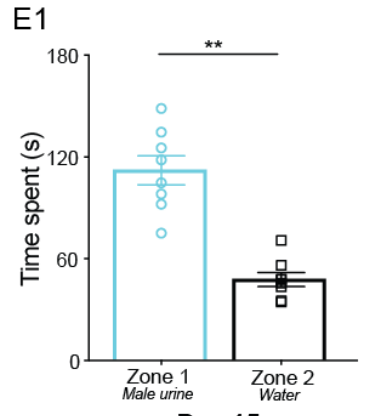

Day 15

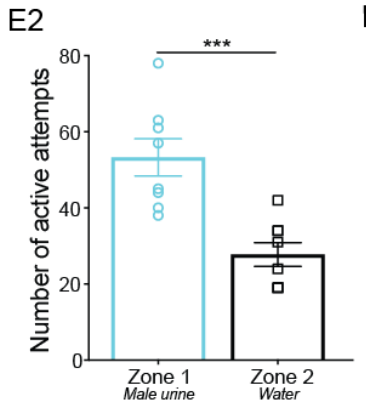

Day 15

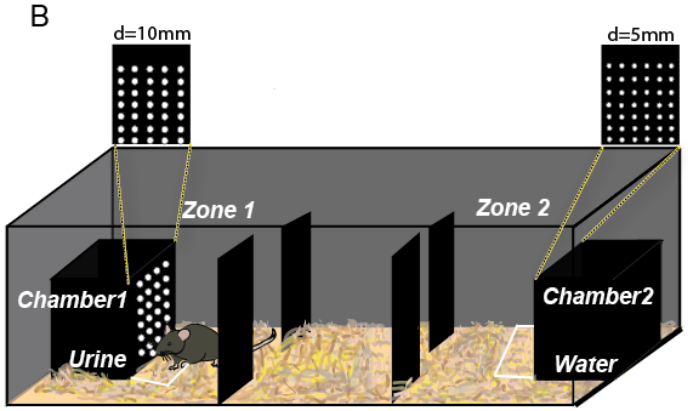

C2

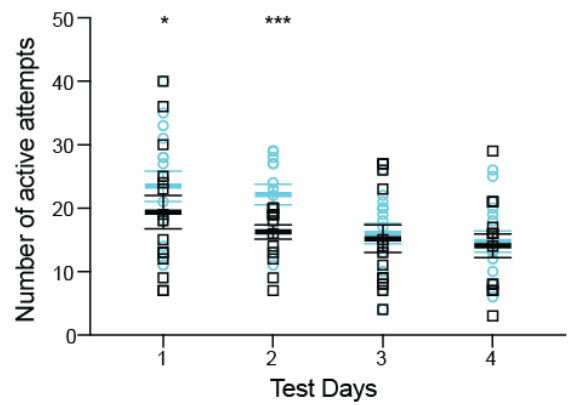

D2

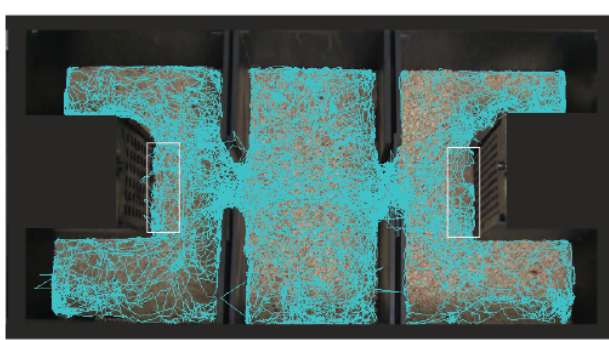

E3

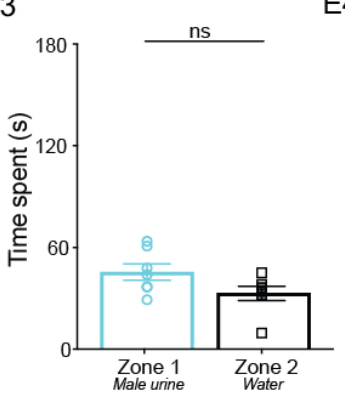

Day 30
E4

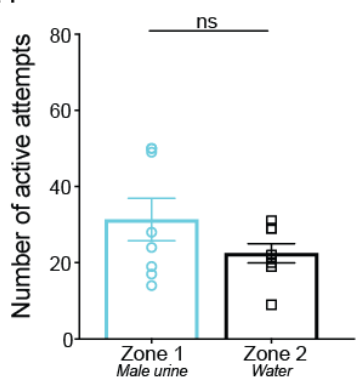

Day 30 
Figure 1. Multimodal associative learning causes long-term memory formation of pheromone locations in female mice.

A. Experimental design of 'multimodal pheromonal learning' paradigm. It involves an initial testing phase of 4 days, training phase of 15 days and the testing of memory at $15^{\text {th }}$ and $30^{\text {th }}$ day post training.

B. Diagrammatic representation of the set-up used for the assay. Female mouse is shown to be sensing the volatiles emanating through the specific diameter holes from the stimulus present in OSP chamber. Perimeter demarcated by white line represents the zone where time spent by mouse is calculated using the EthoVision software by tracking the 'nose-point' of the animal.

C1. Similar time spent near OSP chamber (blue bar) v/s NS chamber (black bar) on day 1, 3 and 4 of testing phase. Increased time spent on day 2 indicates emerging preference towards OSP which, however, declines as the days of exploration progress. ( $p>0.05, F=4.34$ Repeated Measures (RM) Two-way ANOVA; Bonferroni's multiple comparison test, $p<0.05$ for Day $2, p=0.1$ for Day $1, \mathrm{p}>0.9$ for Day 3 and Day $4 ; \mathrm{n}=13$ mice) Urine and water stimuli were replaced each day during testing and training phase.

C2. Similar number of active attempts on plate guarding OSP chamber v/s NS chamber on day 3 and 4 during initial testing phase. On day 1 and day 2, mice exhibited more number of active attempts manifesting initial preference towards OSP, which was reduced on day 3 and $4(p>0.05, F=4.1$ RM Two-way ANOVA; Bonferroni's multiple comparison test, $p=0.016$ for day $1, p=0.0003$ and day $2, p>0.9$ for day 3 and day $4 ; n=14$ mice)

D1, D2. Tracks recorded using EthoVision software by tracking the 'nose point' of the animal. Visibly more time spent near zone 1 compared to zone 2 in case of $15^{\text {th }}$ day track (D1) while equal time spent for both the zones in case of $30^{\text {th }}$ day track (D2) is seen. The area demarcated is used for calculating time spent near the two chambers.

E1. More time was spent near OSP chamber than NS chamber on Day 15 post training period ( $p=0.007$, Wilcoxin matched pairs signed rank test). No stimuli (urine/water) were kept in the chambers.

E2. Number of active attempts on plate guarding OSP chamber was higher than on NS chamber on Day 15 post training period ( $p=0.0004$, Paired two-tailed student's t-test). No stimuli (urine/water) were kept in the chambers.

E3. Similar amount of time was spent near OSP chamber and NS chamber on Day 30 post training period $(p>0.1$, Wilcoxin matched pairs signed rank test, $n=$ 8 mice). No stimuli (urine/water) were kept in the chambers.

E4. Similar number of active attempts were made on plate guarding OSP chamber and NS chamber on Day 30 post training period $(p>0.1$, Wilcoxin matched pairs signed rank test, $n=8$ mice). No stimuli (urine/water) were kept in the chambers.

student's t-test) after the multi-modal training was carried out. However, we did not observe any preference when the $30^{\text {th }}$ day memory was analyzed (Figure 1E3: $p>$ 0.05 for time spent and Figure 1E4: $p>0.1$ for number of active attempts, Wilcoxin matched pairs signed rank test) after the training. As mice were not exposed to the stimuli for an extended period of 30 days, it possibly led to extinction of preferential memory for the attractive stimulus. This result is in line with other studies, which have shown that female mice can remember the scent location up to two weeks after a single exposure to the urine scent (Roberts et al., 2014; Roberts et al., 2012). However, in their study, the reported memory was dependent on the spatial cue based learning. 
To systematically investigate if whisker system is being utilized as a result of repeated snout insertions into the plate with holes of specific diameter during the training phase, we carried out whisker sensory deprivation in another set of mice (Group 2, see Methods). In this experiment, whiskers were trimmed and an anesthetic gel was applied on the snout of the mouse during the training phase of 15 days so as to prevent the association of volatiles emanating from the stimulus kept inside the chambers guarded with plates of specific hole diameters. Application of anesthetic gel was done each day, 15 minutes before putting the mouse in the setup for the training period. Towards end of this recovery period we observed normal mobility with all animals tested. The mice were then put in the multi-modal pheromonal learning set-up. After finishing the training, the $15^{\text {th }}$ day pheromone location memory was tested in these mice. Whiskers were not trimmed after the training period and all mice had regrown whiskers on the day of memory investigation. The memory was significantly reduced in the whisker deprived group compared to whisker intact group indicating the involvement of whisker system in facilitating the multimodal learning and thereby the memory formation for pheromone locations (Figure 2A2: $p<0.0001, F=18.69$ Ordinary One-way ANOVA; Bonferroni's multiple comparison test, $p=0.0008$ for time spent and Figure 2B2: $p<$ $0.0001, F=15.27$ Ordinary One-way ANOVA; Bonferroni's multiple comparison test, $p=0.006$ ). Indeed, we did not observe difference in the time spent in front of the OSP chamber v/s NS chamber (Figure S2A1: $p>0.1$, Paired two-tailed student's ttest) and the number of active attempts into the holes on both the chambers (Figure S2A2: $p>0.1$, Paired two-tailed student's t-test) suggesting that preference for attractive, opposite sex pheromonal stimuli was impaired for this group.

To study if the preference shown by female mice were specific to the opposite sex pheromones, we carried out multi-modal training paradigm with "non-attractive", same-sex pheromones using another set of female mice (Group 3, see methods). On testing the memory shown by females towards female urine and bedding, we observed significantly lower response compared to the preference shown by Group 1 mice towards male pheromones (Figure 2A2: B2 $p<0.0001$ for both parameters, Ordinary One-way ANOVA, Bonferroni's multiple comparison test). This indicates the robustness of association and the specificity in memorizing the location of opposite sex pheromones using our multi-modal learning paradigm.

The differential responses shown by animals towards same and opposite sex pheromones can arise as a result of differential activity of sex -selective neurons present in the AOB of female mice. This sex-selectivity is based on the varying extent of lateral inhibition in the circuitry and can, thus, differentially regulate the pathway required for sensing male urine v/s female urine within the $A O B$ (Hendrickson et al., 2008). However, as our paradigm requires multi-modal form of learning and memory, it could be possible that the differential response to these stimuli (male urine v/s female urine) could also arise as a result of activity changes occurring in higher centers. The importance of tactile cues along with the pheromones leading to accelerated puberty in female mice suggests involvement of somatosensory regions (Bronson and Maruniak, 1975). A recent study indicates that an initial sexual experience can lead to gain in the population of excitatory Scnn1a +ve neurons in layer 4 of genital cortex in pubertal female mice (Sigl-Glockner et al., 2019). As we do observe a vivid association involving whisker pathway, which leads 
to a robust memory of pheromones, it will be critical to study the underlying structural plasticity in sensory cortical areas.

The prevalent notion in the field is that human pheromones such as axillary secretions, secretions present in urine, semen, vaginal fluid etc. can be detected by the opposite sex leading to arousal-like state (Brennan and Keverne, 2004; Comfort, 1971; Doty et al., 1975; Doty et al., 1978). Although the presence of functional VNO is highly debated in adult humans (Kouros-Mehr et al., 2001), they can still perceive pheromones, which suggests the ability of pheromone detection by the MOB and the trigeminal system (Doty, 2008). The multi-modal aspect of our assay leading to persistence of preference memory towards urinary pheromones suggests that pheromonal communication is not just a simple stimulus-response system. It involves action from multiple sensory systems to generate a preference and memory of pheromones. This aspect is similar to what is found in humans where the mediation of sexual attraction involves both tactile sense and the attractive pheromonal detection (Verhaeghe et al., 2013). Yet another aspect is the role of repeated active exposure to pheromonal stimuli leading to increased learning in case of the female mice, a similar observation in humans has been seen. Human subjects who were previously insensitive to androstenone, a male pheromone, could indeed sense this odor when a systematic exposure to androstenone was done repeatedly (Wysocki et al., 1989). Active pheromonal learning and memory could thus, lead to persistent enhanced attraction towards a particular mate in case of humans too. However, this notion needs to be systematically studied using monogamous model systems.

Sniffing modulations happen while the animals are engaged in non-pheromonal volatile driven decisions (Bhattacharjee et al., 2019). In our paradigm, animals need to take the decisions, which may involve adopting varying sampling strategies, towards the pheromonal cues critical for their reproductive/social life. Therefore, we investigated their sampling behavior using subsets of female mice utilized for multimodal pheromonal learning assay. We analyzed the sniffing frequencies shown by whisker intact and deprived animals towards the volatile pheromonal cues during brief pheromonal exposure epochs. These mice were head-restrained and urine volatiles were delivered using a custom-built olfactometer. Their sniffing strategies did not vary under different conditions for the time duration we tested for (Figure S3: $p>0.1$ across all groups on four consecutive days, Ordinary One-way ANOVA). This confirms the fact that the reduced memory we observed for the whisker deprived group towards the opposite sex urine and the whisker intact group towards the same sex pheromones is not due to sampling differences, but because of the robustness and specificity of associations formed between different modalities. 
A1

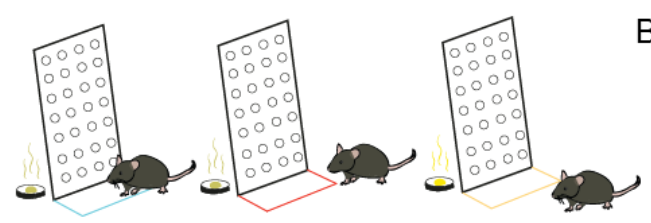

A2

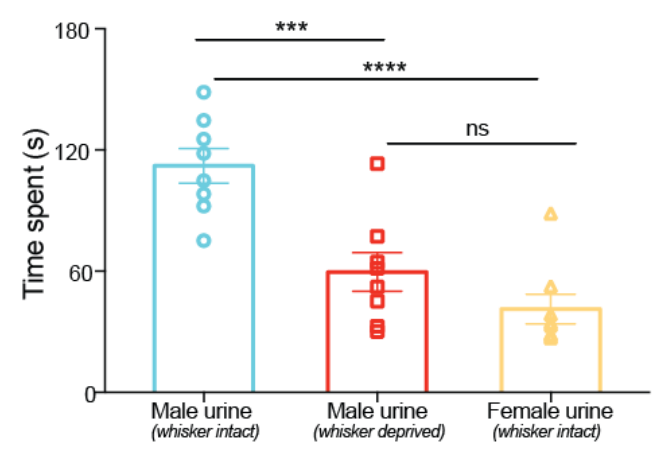

B1

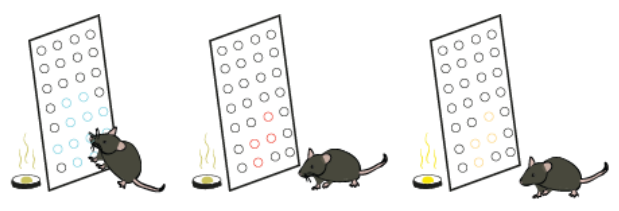

B2

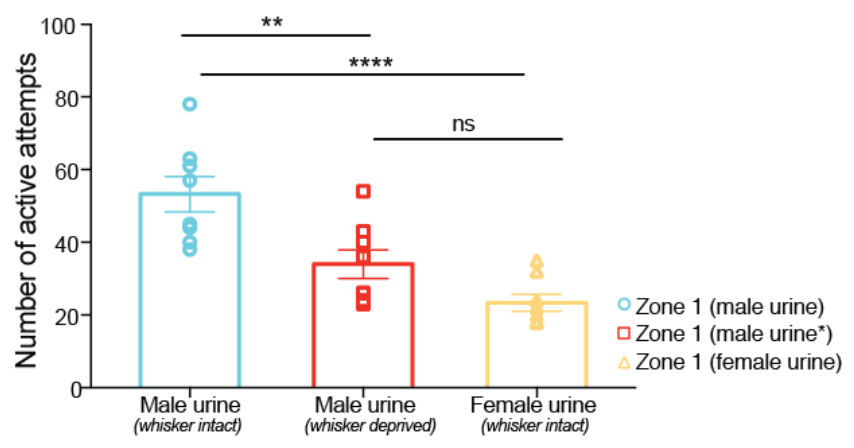

Figure 2. Long-term memory of pheromone location is facilitated by the association formed between olfactory and whisker systems.

A1. Schematic representing a female mouse sampling in front of the plate guarding OSP chamber in the perimeter demarcated by a colored line. Nose point of the mouse snout is tracked for measuring parameter "time spent" by EthoVision software (Blue: whisker intact mouse trained towards male MBU, Red: whisker deprived mouse towards MBU and Yellow: whisker intact mouse towards FBU). Memory was tested in the absence of any stimuli (urine, soiled bedding and water).

A2. Reduced memory was observed for whisker deprived mice trained with male urine and whisker intact mice trained with female urine compared to whisker intact mice trained with male urine on $15^{\text {th }}$ day memory testing $(\mathrm{p}<0.0001, \mathrm{~F}=$ 18.69, Ordinary One-way ANOVA, Bonferroni's multiple comparison test; Male urine v/s Male urine*: $p=0.0008$, Male urine v/s Female Urine: $p<0.0001$, Male urine $^{*} v / \mathrm{s}$ Female urine: $p>0.1, n=8$ mice for all groups) ( ${ }^{*}$ whisker deprived).

B1. Schematic representing a female mouse making active attempts by poking its snout multiple times into the holes of a particular diameter on plate guarding OSP chamber. Color of the holes circumference depict the experimental condition; blue: whisker intact group trained towards male urine; red: whisker deprived group towards male urine and yellow: whisker intact group towards female urine. Memory was tested in the absence of any stimuli (urine, soiled bedding and water).

B2. Reduced number of active attempts were observed for whisker deprived mice trained with male urine and whisker intact mice trained with female urine compared to whisker intact mice trained with male urine on $15^{\text {th }}$ day memory testing $(p<0.0001, F=15.27$, Ordinary One-way ANOVA, Bonferroni's multiple comparison test; Male urine v/s Male urine*: $p<0.01$, Male urine v/s Female Urine: $p<0.0001$, Male urine ${ }^{*} \mathrm{v} / \mathrm{s}$ Female Urine: $\mathrm{p}>0.1, \mathrm{n}=8$ mice for all groups) ( ${ }^{*}$ whisker deprived).

In order to assess the role of pre-cortical region, the olfactory bulb in governing the mediation of extensive learning and memory for male urine pheromones in our assay, we investigated the modulation in adult neurogenesis of MOB and AOB. We conducted immunohistochemical analysis of Bromodeoxyuridine (BrdU) expression 
through the learning phase. BrdU injections were carried out on a single day, i.e. the $4^{\text {th }}$ day of the initial testing phase. Mice were then trained for 15 days in the assay and were kept in their home cages without exposure to the trained pheromonal stimuli for the next 15 days. Just after checking the pheromonal memory on $30^{\text {th }}$ day after the injections ( $15^{\text {th }}$ day post training) after the BrdU injection, the quantification of BrdU expressing cells was carried out. We observed increased number of BrdU positive cells in the MOB of trained female mice exposed to male urine compared to the female mice exposed to female urine in our assay. (Figure 3 A4: $p<0.05$, Unpaired two-tailed student's t-test) The multimodal aspect of pheromonal learning paradigm allowed the mice to sense the volatiles repeatedly through the holes for a period of 15 days during the training. During the memory testing, we observed these mice making more number of active attempts on the chamber containing male urine, thus, displaying the memory of volatiles as compared to the control group exposed to female urine (Figure 2B2: $p<0.0001$ One-way ANOVA; Bonferroni's multiple comparison test). As MOB is known to process the signals from urine volatiles, it is possible that the intact memory for volatiles emanating from opposite sex urine could be aided by increased MOB neurogenesis. It is known that learning of volatile odor discriminations lead to increased MOB neurogenesis (Lledo et al., 2006). Indeed, in our assay, mice are involved in an active learning of associating pheromones with the hole size rather than 'exposure alone' studies (Brennan and Keverne, 2004).

In the quantification of $A O B$ adult neurogenesis, we did not observe any increase in the number of BrdU positive cells (Figure 3B4, $p>0.1$, Unpaired two-tailed student's t-test). A study shows reduced number of adult-born neurons in AOB being activated (c-fos/BrdU co-localized) when checked 7 days after the exposure to male soiled bedding (Oboti et al., 2011). This indicates that the absence of stimuli can possibly bring back the AOB neurogenesis to a basal level. Also, as the functional value of increased $A O B$ neurogenesis is mostly in it's involvement in having a successful mating, it is expected that when the pheromones are absent, AOB neurogenesis would drop down to basal level. In our case, we check for BrdU positive cells 15 days after the training period, i.e.15 days of no exposure to male urine and bedding and thus, we observe similar $A O B$ neurogenesis levels in mice exposed to male urine and female urine (Figure 3B4: $p>0.05$, Mann-Whitney test). It has also been shown that maximum number of $A O B$ new-born BrdU-positive granule cells are activated at their 2-3 weeks of cell age upon pheromonal exposure (Oboti et al., 2009). However, our quantification was beyond this period, which may explain similar AOB neurogenesis levels between the groups tested. Further, we need to systematically investigate the modulatory nature of $A O B$ neurogenesis as well as the turnover rates under sensory enriched conditions.

As social olfactory recognition and memory can be modulated by oxytocin (Young et al., 2001) and vasopressin neuropeptides (Lukas and Neumann, 2013), it is worthwhile to investigate the role of these peptides in governing the multi-modal pheromonal location learning behavior. In olfactory system, oxytocin receptors are primarily present in granule cell layer (GCL) (Ferris et al., 2015), anterior olfactory nucleus (AON) and piriform cortex (PC) (Mitre et al., 2016). The signaling through these receptors allows the circuit to achieve stimulus specificity and increased signal-to-noise ratio for odorants (Oettl et al., 2016). Oxytocin is shown to be indispensable in case of associative social learning but not non-social learning paradigms by mediating its action in PC (Choe et al., 2015). As PC pyramidal neurons undergo activity-dependent changes in case of whisker deprivation (Ye et 
al., 2012), it would be interesting to look how processing through oxytocin in PC can be modulated in case of whisker intact v/s whisker deprived group trained on multimodal pheromonal location learning assay.

A1

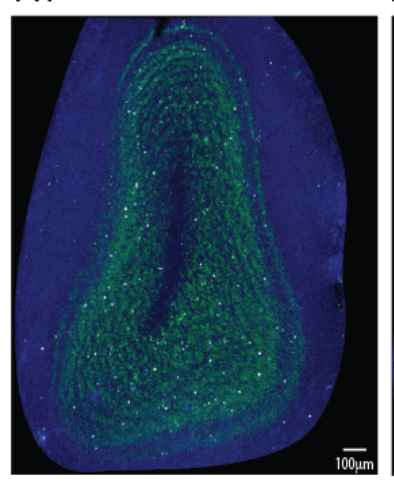

B1

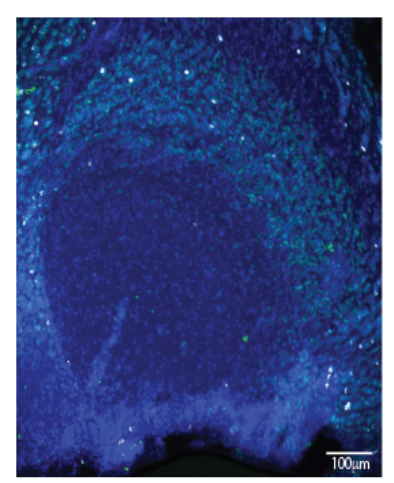

A2

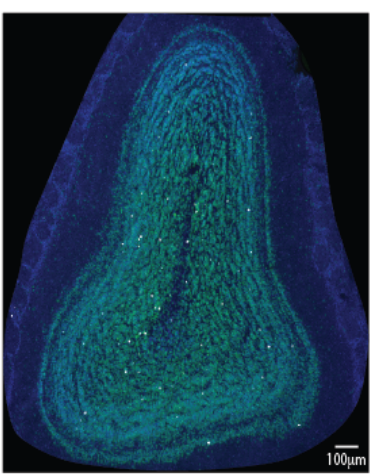

B2

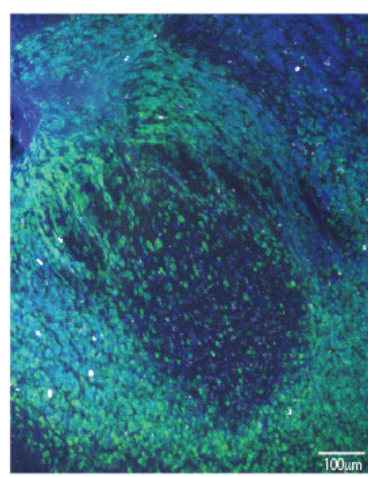

A3

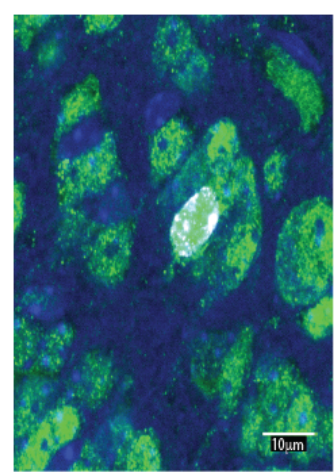

B3

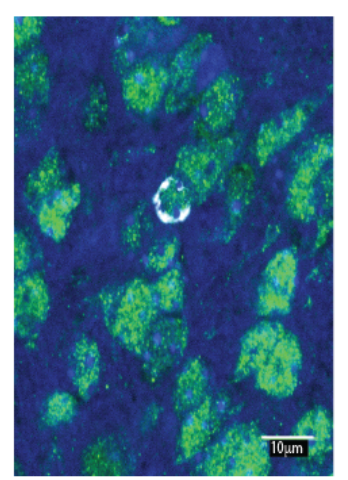

A4

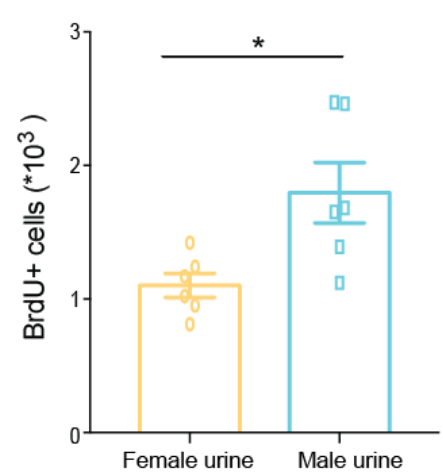

B4

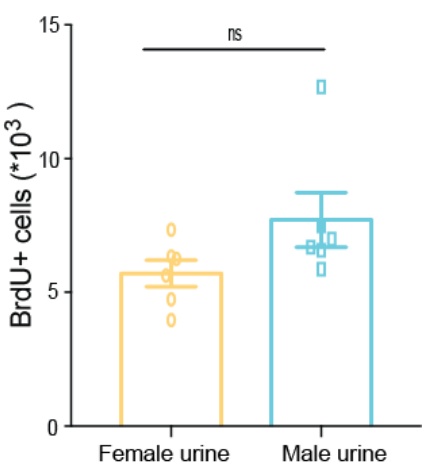

Figure 3. Multi-modal pheromonal location learning causes an increase in MOB neurogenesis

A1. A $50 \mu \mathrm{m}$ section of main olfactory bulb of experimental female mouse exposed to male urine and bedding. Scale bar $=100 \mu \mathrm{m}$, Blue- DAPI, GreenNeuN, Gray- BrdU

A2. A $50 \mu \mathrm{m}$ section of main olfactory bulb of experimental female mouse exposed to female urine and bedding. Scale bar $=100 \mu \mathrm{m}$, Blue- DAPI, GreenNeuN, Gray- BrdU

A3. A $100 x$ image of double NeuN-BrdU positive cell in main olfactory bulb. Scale bar $=10 \mu \mathrm{m}$

A4. Increased number of BrdU-positive cells was observed in main olfactory bulb in female mice trained with male urine compared to female mice trained with female urine in preference assay checked on day 15 after training $(p<0.05$, unpaired two-tailed student's t-test, $\mathrm{N}=3$ mice, $\mathrm{n}=6 \mathrm{MOBs}$ )

B1. 10x image of accessory olfactory bulb experimental female mouse exposed to male urine and bedding. Scale bar $=100 \mu \mathrm{m}$.

B2. 10x image of accessory olfactory bulb experimental female mouse exposed to female urine and bedding. Scale bar $=100 \mu \mathrm{m}$.

B3. A 100x image of double NeuN-BrdU positive cell in accessory olfactory bulb. Scale bar $=10 \mu \mathrm{m}$.

B4. Similar number of BrdU-positive cells was observed in accessory olfactory bulb of female mice trained with male urine compared to female mice trained with female urine in preference assay checked on day 15 after training $(p>0.05$, Mann-Whitney test, $\mathrm{N}=3$ mice, $\mathrm{n}=6 \mathrm{AOBs}$ ) 
So far, we observed the pheromonal location learning and increased MOB neurogenesis in case of female mice exposed to male urine and bedding. We further probed if the positive neural modulation achieved in MOB via increased neurogenesis is bringing about differences in MOB dependent odor discrimination tasks. To this end, we carried out go/no-go odor discrimination paradigm (Bhattacharjee et al., 2019) to investigate modulation of discrimination learning and memory of non-pheromonal volatiles in Whitten effect induced female mice (Figure $4 \mathrm{~A}, \mathrm{~B})$. Cl v/s EU odor pair training was carried out for both the groups before Whitten effect was induced in experimental group to ensure that the learning pace is similar (Figure 4C: $\mathrm{Cl}$ v/s EU Ordinary Two-way ANOVA, Bonferroni's multiple comparison test, $p>0.05$ for all data points in learning curve). Induction and synchronization of estrous cycle, i.e. Whitten effect was achieved in a group of female mice by exposing them directly to male urine and bedding. Once the synchronization was achieved, these mice were trained on an odor-reward association task using the go/no-go odor discrimination paradigm. This was done to observe if $\mathrm{MBU}$ exposed mice can achieve quicker learning on an odor-reward association task as compared to mice who were just exposed to their female conspecifics in their cage. We did not find any differences in the learning pace for various odor pairs (Figure 4C: AA v/s EB: $p>0.1, F=0.12 ; B Z$ v/s NN: $p>0.1, F=$ 1.43; HX v/s PN: $p>0.1, F=0.19$; Ordinary Two-way ANOVA, Bonferroni's multiple comparison test, $p>0.05$ across all data points for all learning curves). Individual day accuracies were also plotted for AA v/s EB odor pair to compare the day-wise accuracy level changes between the experimental and control groups for 4 continuous days (as the estrous cycle was of four days length in synchronized group) during the discrimination training days (Figure 4E: $p>0.1, F=0.49$, Ordinary Two-way ANOVA; Bonferroni's Multiple comparison test, $p>0.9$ across all data points). Discrimination accuracy levels were independent of the estrous stages mice were in. We also checked if the memory for one of the odor pairs (AA v/s EB) was enhanced when checked one month later after the discrimination training. MUB exposure leading to estrous synchronization did not lead to changes in MOB dependent odor discrimination behavior (Figure 4F: $p>0.1$, Mann-Whitney test). These results indicate least influence from the estrous stages on the odor discrimination learning and memory at least with the paradigm we used. 
A1

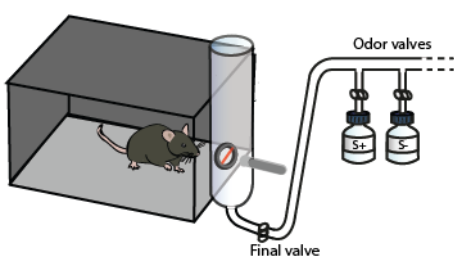

IR beam is maintained and trial is not begun until mouse enters the sampling port

A2

S+ stimulus presentation (Go)

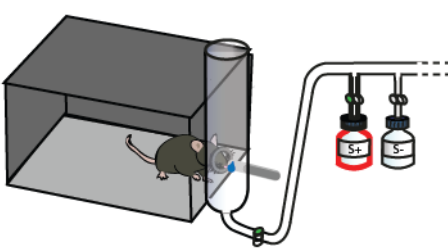

Mouse stays in and gets the reward for $\mathrm{S}+$ odor

A3

S- stimulus presentation (No-Go)

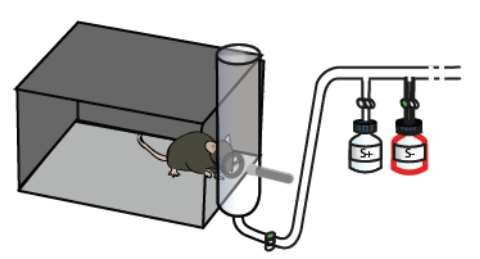

Mouse retracts its head for S- odor
B
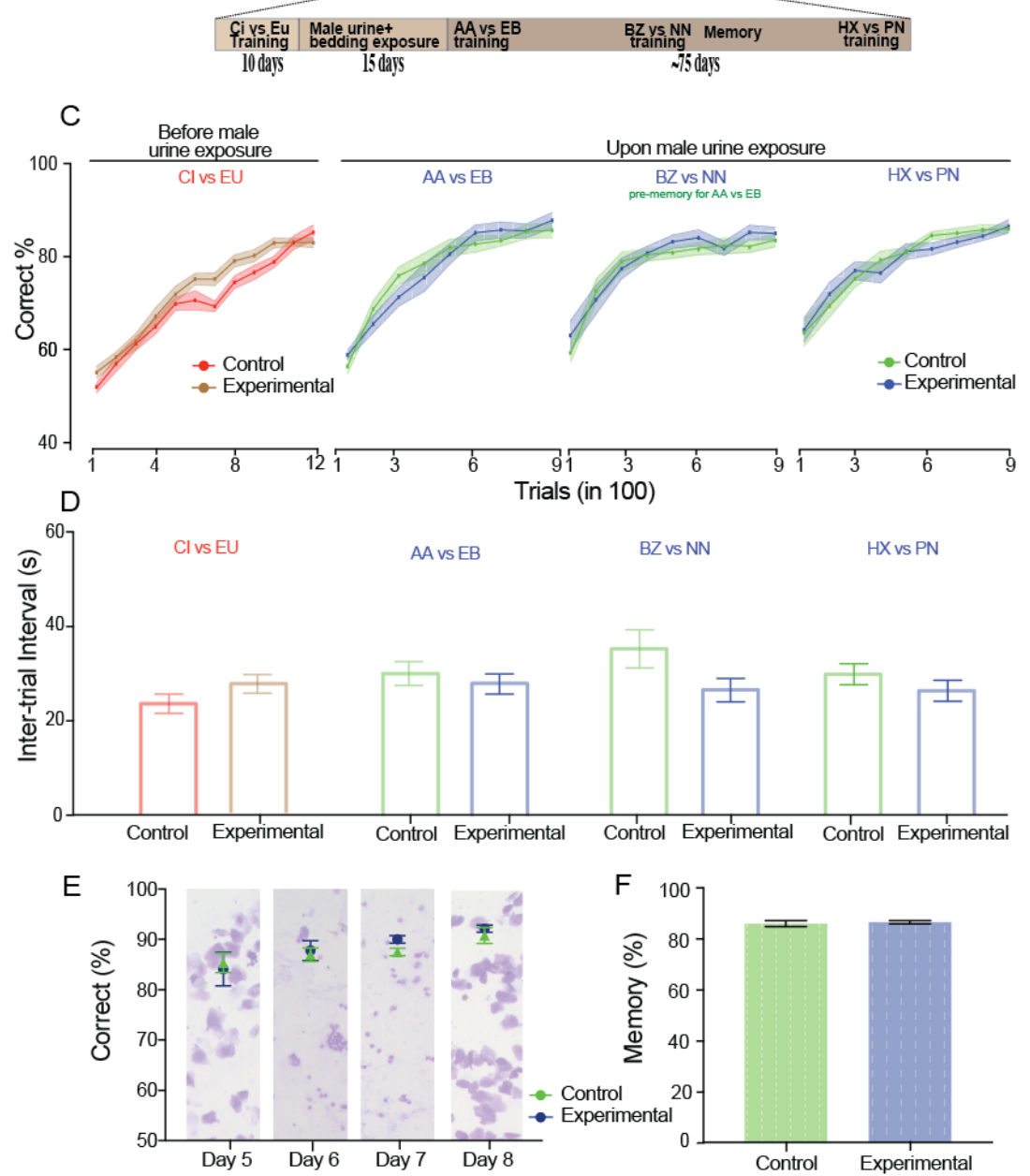

Figure 4. Non-pheromonal volatile discrimination learning and memory is not affected by Whitten effect

A1, A2, A3. Schematic of go/no-go odor discrimination paradigm in a freely moving custom-made olfactometer indicating the sequence of events in a rewarded (A2) v/s unrewarded trial (A3) for a trained mouse.

B. Experimental design for the go/no-go associative olfactory training for MBU exposure and no-exposure female mice groups to investigate their olfactory discrimination learning and memory

C. Learning curves depicting accuracy of discrimination across different odor pairs before MBU and after MBU exposure, shown as percent correct choices of 100 trials. Before Whitten effect was induced, mice from both the groups were trained for $\mathrm{Cl}$ vs EU to ensure similar learning efficiencies (Ordinary Two-way ANOVA, Bonferroni's multiple comparison test, $p>0.05$ for all data points in the learning curve). No difference in the pace of learning across different odor pairs was observed after Whitten effect was induced (AA vs EB: $p>0.1, F=0.12$; BZ vs $\mathrm{NN}$ : $p>0.1, \mathrm{~F}=1.43$; $\mathrm{HX}$ vs $\mathrm{PN}$ : $p>0.1, \mathrm{~F}=0.19$; Ordinary Two-way ANOVA, Bonferroni's multiple comparison test, $p>0.05$ across all data points for all learning curves, $n=11-15$ mice for both groups across odor pairs).

D. Inter-trial interval for both groups across different odor pairs. It gives an indication of motivation of carrying out the trials, i.e. motivation is similar in both the groups. Cl vs EU: $p=0.2$, Mann-Whitney test; AA vs EB: $p=0.42$, BZ vs $N N: p=0.06, H X$ vs PN: $p=0.26$, Unpaired two-tailed student's t-test, $n=11-15$ mice for both groups across odor pairs). 
E. Average discrimination accuracy values during four consecutive days of AA vs EB training done after the induction of Whitten effect. On each of these days, the accuracy was similar between experimental and control group. Vaginal cytology in background for each days on the plot depicts induction and synchronization of estrous cycle for experimental group (estrous: day 5, metestrous: day 6, diestrous: day 7 and proestrous: day 8$)(p>0.1, F=0.49$, Ordinary Two-way ANOVA; Bonferroni's Multiple comparison test, $p>0.9$ across all data points, $n=$ 7 mice)

F. Memory for AA vs EB odor pair checked one month after the odor pair learning was performed by mice. Memory was similar across both the groups. ( $p$ $>0.1$, Mann-Whitney test, $n=13$ for both groups)

Many reports suggest mammalian female olfactory system is more effective as compared to males, part of which is dependent on the endocrine status (Doty and Cameron, 2009; Schneider et al., 1958). Our study rules out the possibility for the estrous phase of a female mouse modulating the olfactory discrimination ability of mice. The learning pace across different odor pairs is independent of whether the estrous cycle is induced and synchronized or not (AA v/s EB, BZ v/s NN, HX v/s PN: $p>0.1$, Ordinary Two-way ANOVA). Therefore, our results demonstrate that the probable sex-dependent changes in the behavioral readouts depend on the type of paradigm and the complexity of task employed.

Our study shows that olfactory discrimination of non-pheromonal volatile odors is independent of the physiological and neurogenesis related changes that occur upon male urine and bedding exposure. With respect to socio-sexual preference, female mice could exhibit enhancement of pheromonal location memory when more than one sensory system is mediating the associative learning.

\section{Author Contributions}

N.A. and M.P. carried out the study conceptualization and experimental design. M.P. and S.M. performed pheromonal location preference behavioral experiments. M.P. analysed the data acquired from pheromonal location preference behavior. M.P., S.M. and U.D. performed and analyzed non-pheromonal volatile discrimination experiments. M.P. acquired and analyzed immunohistochemistry data. N.A. and M.P. wrote the manuscript with comments from S.M.

\section{Acknowledgements}

We thank Thomas Kuner, N.K. Subhedar and Laboratory of Neural Circuits and Behaviour (LNCB) members for fruitful discussions. We thank staff of National Facility for Gene Function in Health and Disease (NFGFHD) and IISER BiologyLeica microscopy facility for the technical support. This work was supported by the Wellcome Trust - DBT India Alliance intermediate grant (IA/I/14/1/501306 to N.A.), IISER-Pune Fellowship (M.P.) and CSIR Fellowship (S.M.). 


\section{Supplementary Figures}
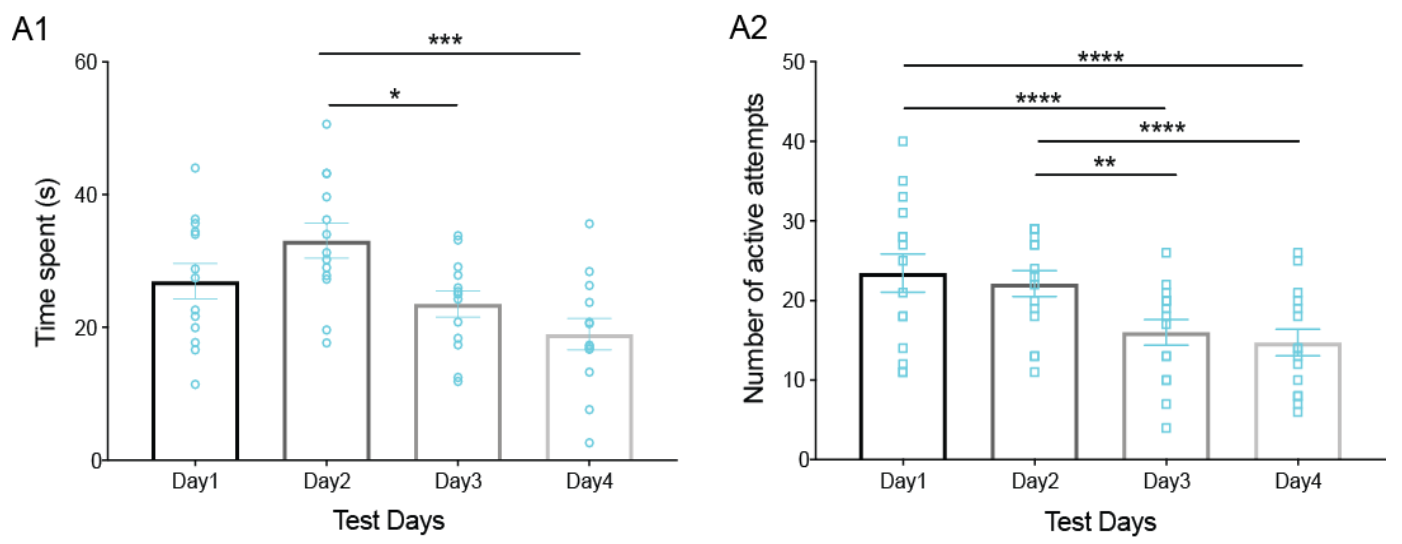

Supplementary Figure S1. Decayed preference for pheromone locations in whisker-intact female mice exposed to a choice of male urine and neutral stimulus

A1. Time spent near OSP chamber containing male urine and soiled bedding by whisker intact female mice. Decayed preference towards this chamber was observed during the initial testing phase $(p<0.01, F=6.3$, RM One-way ANOVA; Bonferroni's multiple comparison test, $p=0.04$ for day 2 v/s day 3 and $p$ $=0.001$ for day $2 \mathrm{v} / \mathrm{s}$ day $4 ; \mathrm{p}>0.1$ for all other comparisons, $\mathrm{n}=13 \mathrm{mice}$ ).

A2. Number of active attempts towards OSP chamber (male urine) during the initial 4 days of testing phase. Mice showed lower number of nose pokes on day 3 and 4 ( $p<0.0001, F=16.3$, RM One-way ANOVA; Bonferroni's multiple comparison test, $p<0.0001$ for day $1 \mathrm{v} / \mathrm{s}$ day 3,4 and $p<0.001$ day $2 \mathrm{v} / \mathrm{s}$ day 3 , $4 ; p>0.9$ for all other comparisons, $n=14$ mice).
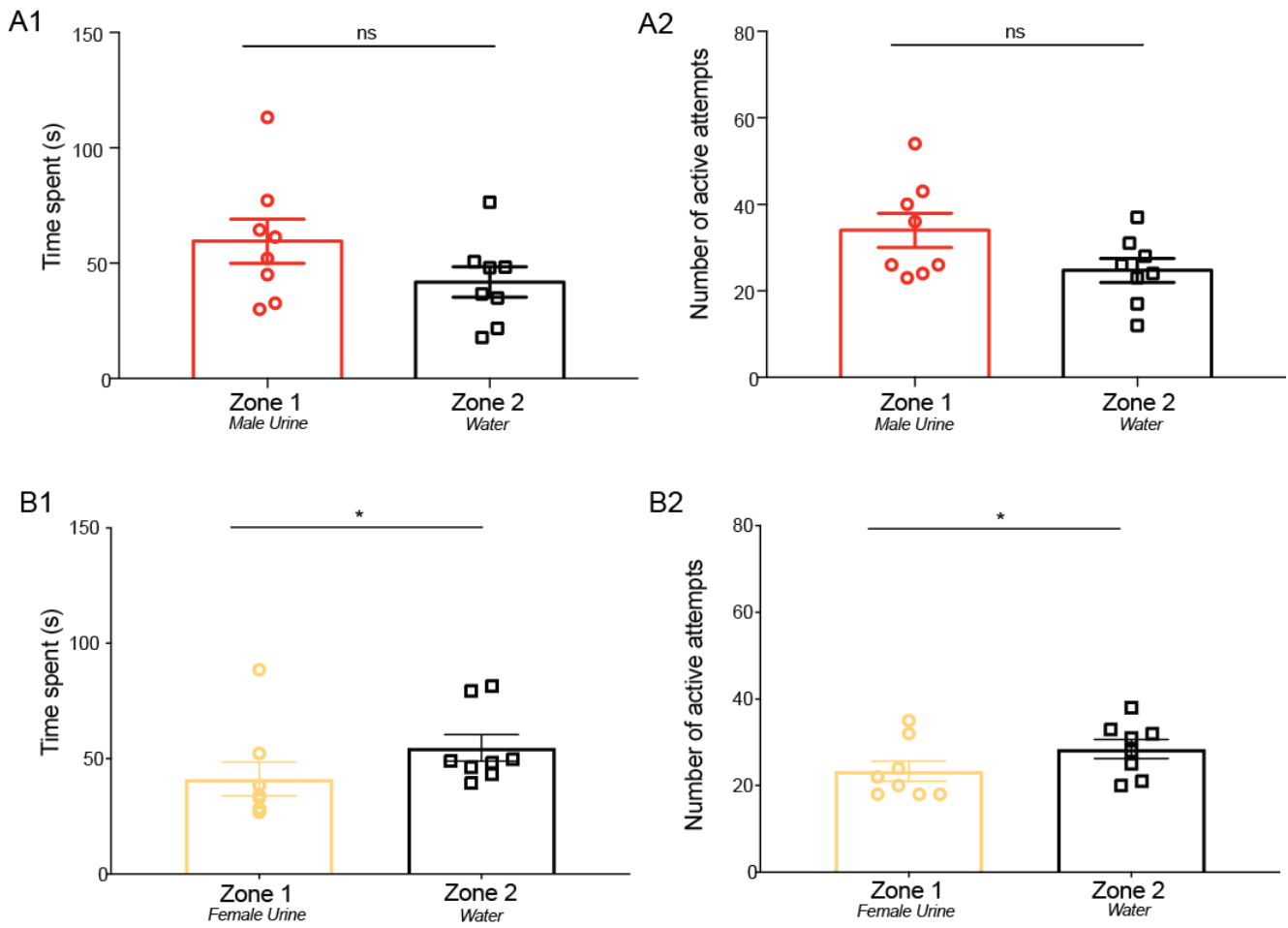

Supplementary Figure S2. Similar sampling behavior shown towards pheromonal and neutral stimuli. 
A1, A2. No preference for OSP as indicated by similar time spent and number of active attempts for whisker deprived female mice on Day $15^{\text {th }}$ memory phase. The mice were trained with male urine and bedding versus water. $(p>0.1$ for time spent and for number of active attempts, Paired two-tailed student's t-test, $n$ $=8$ mice)

B1, B2. Decreased preference for female urine as indicated by increased time spent and number of active attempts on NS chamber for whisker intact female mice on Day $15^{\text {th }}$ memory phase. The mice were trained with female urine and bedding versus water. $(p=0.039$ for time spent and number of active attempts, Wilcoxin matched pairs signed rank test, $\mathrm{n}=8$ mice)

A

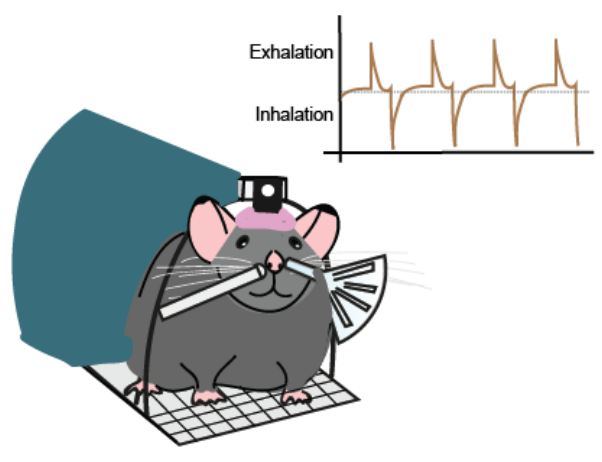

B

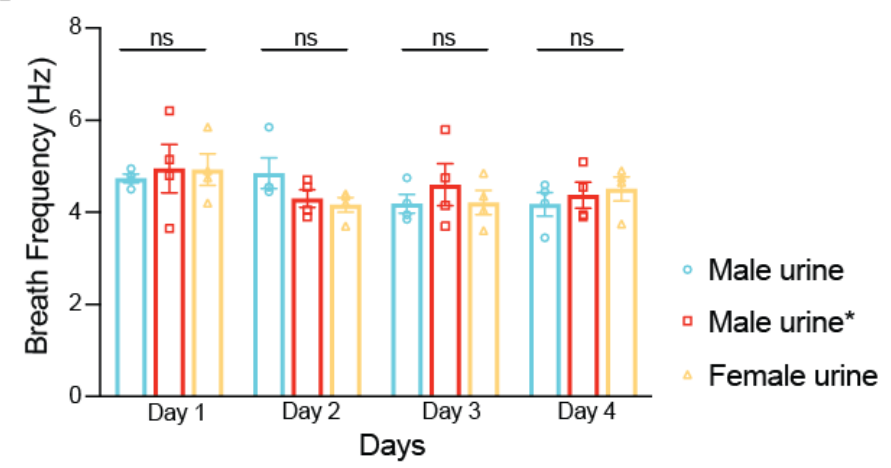

Supplementary Figure S3. Similar sniffing frequency for sampling pheromones under whisker intact and deprived conditions

A. Schematic of a female mouse head-post implanted on the head-restrained setup. Breathing is recorded using air-pressure sensor placed near one nostril (on left nostril in depiction) and delivering the stimulus (male urine or female urine volatiles) pseudorandomized with filtered air presentations from a nozzle (right side). Inset: Representative Inhalation/Exhalation pattern of a mouse breathing during stimulus delivery.

B. Breath frequency of three groups of female mice (blue: whisker intact sensing male urine volatiles, red: whisker deprived, anesthetic applied sensing male urine volatiles and yellow: whisker intact sensing female urine volatiles) recorded across four consecutive days (day $1: p=0.9, F=0.1$; day $2: p>0.1, F=2.2$; day $3: p=$ $0.6, F=0.58$; day $4: p=0.7, F=0.40$; Ordinary One-way ANOVA, Bonferroni's multiple comparison test, $\mathrm{p}>0.1, \mathrm{n}=4$ mice for all groups) (Male urine* ${ }^{*}$ whisker deprived group/Group 2). 
A

M01

M02

M03

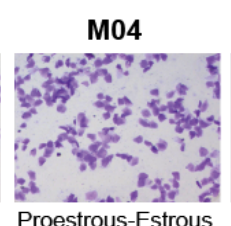

M05
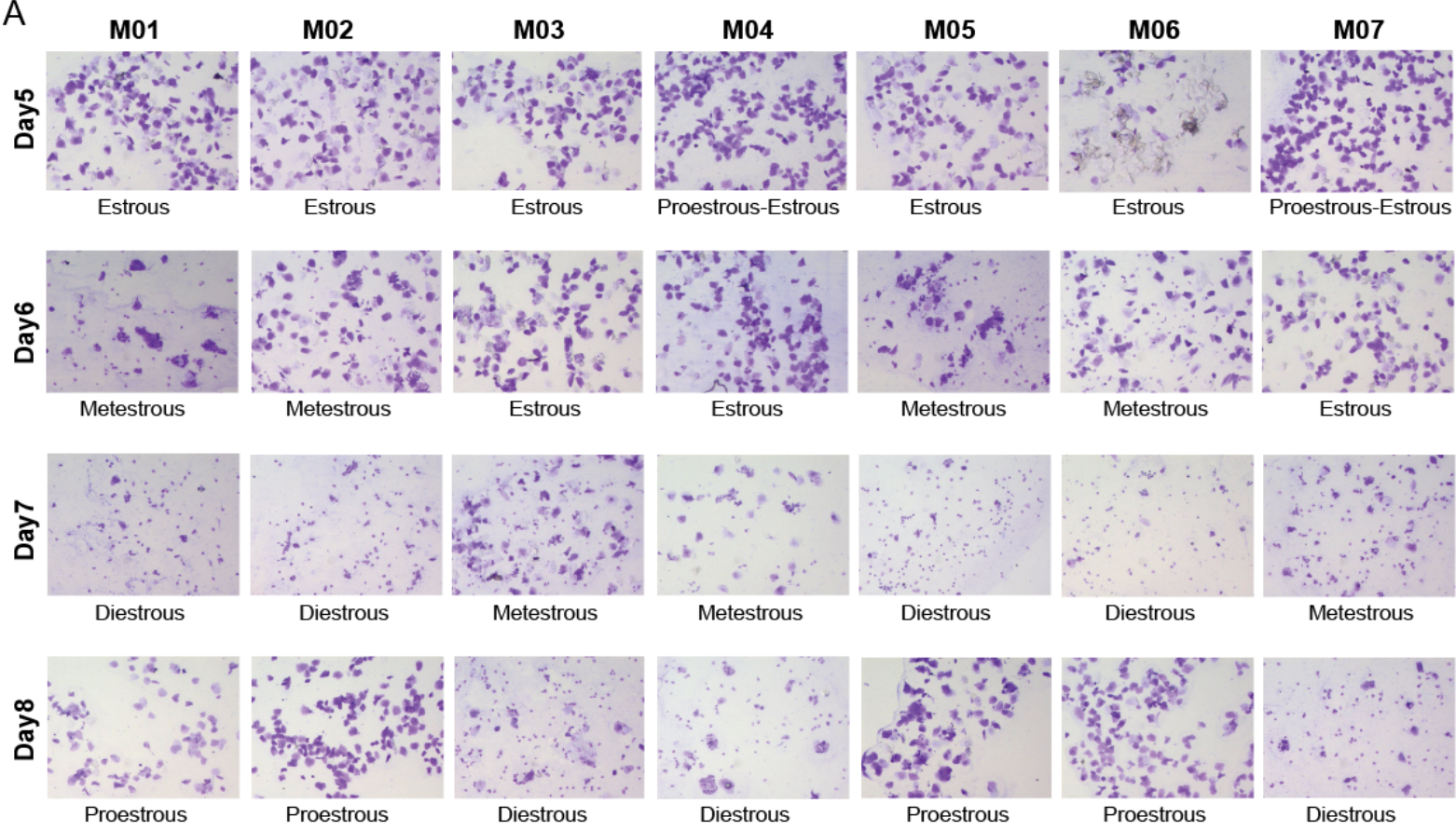

B

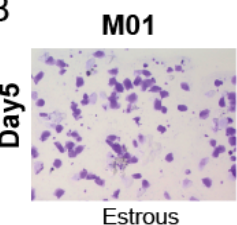

M02
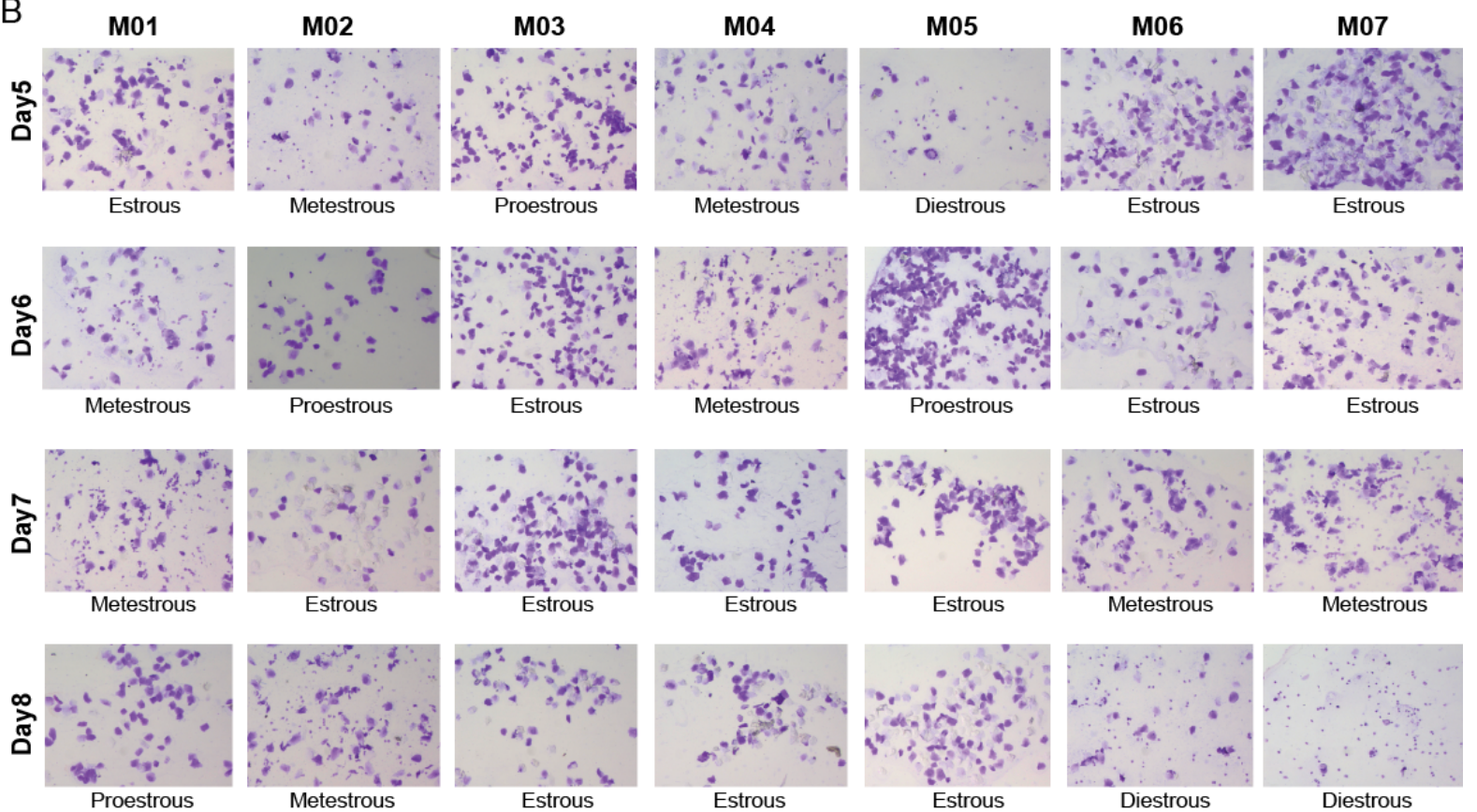

Supplementary figure S4. Whitten effect induction and synchronization in female mice by male pheromonal exposure

A. Whitten effect, i.e. induction and synchronization of estrous cycle continued to occur in experimental female mice during the duration of $A A \mathrm{v} / \mathrm{s} E B$ odor pair training. Subset of mice whose vaginal smears were taken on a daily basis during the training, four consecutive days of induction and synchronization is observed in case of experimental mice (each stage lasts for a day, making it a proper 4 day cycle and follows an expected sequence in cycle from proestrous $\rightarrow$ estrous -> metestrous $\rightarrow$ diestrous).

B. Control group which were not exposed to male urine and soiled bedding exhibited prolonged estrous cycle with one stage lasting $>1$ day and not occurring the proper 
sequence of the cycle, i.e. deviating from proestrous $->$ estrous $->$ metestrous -> diestrous sequence.

\section{References}

Abraham, N.M., Egger, V., Shimshek, D.R., Renden, R., Fukunaga, I., Sprengel, R., Seeburg, P.H., Klugmann, M., Margrie, T.W., Schaefer, A.T., and Kuner, T. (2010). Synaptic inhibition in the olfactory bulb accelerates odor discrimination in mice. Neuron 65, 399-411.

Abraham, N.M., Guerin, D., Bhaukaurally, K., and Carleton, A. (2012). Similar odor discrimination behavior in head-restrained and freely moving mice. PLoS One 7 , e51789.

Abraham, N.M., Spors, H., Carleton, A., Margrie, T.W., Kuner, T., and Schaefer, A.T. (2004). Maintaining accuracy at the expense of speed: stimulus similarity defines odor discrimination time in mice. Neuron 44, 865-876.

Abraham, N.M., Vincis, R., Lagier, S., Rodriguez, I., and Carleton, A. (2014). Long term functional plasticity of sensory inputs mediated by olfactory learning. Elife 3, e02109.

Arakawa, H., Blanchard, D.C., Arakawa, K., Dunlap, C., and Blanchard, R.J. (2008). Scent marking behavior as an odorant communication in mice. Neurosci Biobehav Rev 32, 1236-1248.

Bhattacharjee, A.S., Konakamchi, S., Turaev, D., Vincis, R., Nunes, D., Dingankar, A.A., Spors, H., Carleton, A., Kuner, T., and Abraham, N.M. (2019). Similarity and Strength of Glomerular Odor Representations Define a Neural Metric of Sniff-Invariant Discrimination Time. Cell Rep 28, 2966-2978 e2965.

Bouton, M.E., and Sunsay, C. (2001). Contextual control of appetitive conditioning: influence of a contextual stimulus generated by a partial reinforcement procedure. Q J Exp Psychol B 54, 109-125.

Brennan, P.A., and Keverne, E.B. (2004). Something in the air? New insights into mammalian pheromones. Curr Biol 14, R81-89.

Bronson, F.H., and Maruniak, J.A. (1975). Male-induced puberty in female mice: evidence for a synergistic action of social cues. Biol Reprod 13, 94-98.

Choe, H.K., Reed, M.D., Benavidez, N., Montgomery, D., Soares, N., Yim, Y.S., and Choi, G.B. (2015). Oxytocin Mediates Entrainment of Sensory Stimuli to Social Cues of Opposing Valence. Neuron 87, 152-163.

Comfort, A. (1971). Likelihood of human pheromones. Nature 230, 432-433 passim.

Doty, R.L. (2001). Olfaction. Annu Rev Psychol 52, 423-452.

Doty, R.L. (2008). The olfactory vector hypothesis of neurodegenerative disease: is it viable? Ann Neurol 63, 7-15.

Doty, R.L., and Cameron, E.L. (2009). Sex differences and reproductive hormone influences on human odor perception. Physiol Behav 97, 213-228.

Doty, R.L., Ford, M., Preti, G., and Huggins, G.R. (1975). Changes in the intensity and pleasantness of human vaginal odors during the menstrual cycle. Science 190, 13161318.

Doty, R.L., Orndorff, M.M., Leyden, J., and Kligman, A. (1978). Communication of gender from human axillary odors: relationship to perceived intensity and hedonicity. Behav Biol 23, 373-380.

Dulac, C., and Torello, A.T. (2003). Molecular detection of pheromone signals in mammals: from genes to behaviour. Nat Rev Neurosci 4, 551-562.

Ferris, C.F., Yee, J.R., Kenkel, W.M., Dumais, K.M., Moore, K., Veenema, A.H., Kulkarni, P., Perkybile, A.M., and Carter, C.S. (2015). Distinct BOLD activation profiles following central and peripheral oxytocin administration in awake rats. Front Behav Neurosci 9.

Gratzner, H.G. (1982). Monoclonal antibody to 5-bromo- and 5-iododeoxyuridine: A new reagent for detection of DNA replication. Science $218,474-475$. 
Greer, P.L., Bear, D.M., Lassance, J.M., Bloom, M.L., Tsukahara, T., Pashkovski, S.L., Masuda, F.K., Nowlan, A.C., Kirchner, R., Hoekstra, H.E., and Datta, S.R. (2016). A Family of non-GPCR Chemosensors Defines an Alternative Logic for Mammalian Olfaction. Cell 165, 1734-1748.

Halem, H.A., Cherry, J.A., and Baum, M.J. (1999). Vomeronasal neuroepithelium and forebrain Fos responses to male pheromones in male and female mice. J Neurobiol 39, 249-263.

Hendrickson, R.C., Krauthamer, S., Essenberg, J.M., and Holy, T.E. (2008). Inhibition shapes sex selectivity in the mouse accessory olfactory bulb. J Neurosci $28,12523-$ 12534.

Humphries, R.E., Robertson, D.H., Beynon, R.J., and Hurst, J.L. (1999). Unravelling the chemical basis of competitive scent marking in house mice. Anim Behav 58, 1177-1190.

Hurst, J.L. (1993). The priming effects of urine substrate marks on interacgtions between male house mice, Mus musculus domesticus Schwarz \& Schwarz. Animal Behaviour $45,55-81$.

Ichikawa, M. (2003). Synaptic mechanisms underlying pheromonal memory in vomeronasal system. Zoolog Sci 20, 687-695.

Isogai, Y., Si, S., Pont-Lezica, L., Tan, T., Kapoor, V., Murthy, V.N., and Dulac, C. (2011). Molecular organization of vomeronasal chemoreception. Nature 478, 241-245.

Kimoto, H., Haga, S., Sato, K., and Touhara, K. (2005). Sex-specific peptides from exocrine glands stimulate mouse vomeronasal sensory neurons. Nature 437, 898-901.

Kouros-Mehr, H., Pintchovski, S., Melnyk, J., Chen, Y.J., Friedman, C., Trask, B., and Shizuya, H. (2001). Identification of non-functional human VNO receptor genes provides evidence for vestigiality of the human VNO. Chem Senses 26, 1167-1174.

Liberles, S.D., and Buck, L.B. (2006). A second class of chemosensory receptors in the olfactory epithelium. Nature 442, 645-650.

Lledo, P.M., Alonso, M., and Grubb, M.S. (2006). Adult neurogenesis and functional plasticity in neuronal circuits. Nat Rev Neurosci 7, 179-193.

Lukas, M., and Neumann, I.D. (2013). Oxytocin and vasopressin in rodent behaviors related to social dysfunctions in autism spectrum disorders. Behavioural Brain Research 251, 85-94.

Mitre, M., Marlin, B.J., Schiavo, J.K., Morina, E., Norden, S.E., Hackett, T.A., Aoki, C.J., Chao, M.V., and Froemke, R.C. (2016). A Distributed Network for Social Cognition Enriched for Oxytocin Receptors. Journal of Neuroscience 36, 2517-2535.

Moore, J.D., Deschenes, M., Furuta, T., Huber, D., Smear, M.C., Demers, M., and Kleinfeld, D. (2013). Hierarchy of orofacial rhythms revealed through whisking and breathing. Nature 497, 205-210.

Oboti, L., Savalli, G., Giachino, C., De Marchis, S., Panzica, G.C., Fasolo, A., and Peretto, P. (2009). Integration and sensory experience-dependent survival of newly-generated neurons in the accessory olfactory bulb of female mice. Eur J Neurosci 29, 679-692.

Oboti, L., Schellino, R., Giachino, C., Chamero, P., Pyrski, M., Leinders-Zufall, T., Zufall, F., Fasolo, A., and Peretto, P. (2011). Newborn interneurons in the accessory olfactory bulb promote mate recognition in female mice. Front Neurosci 5, 113.

Oettl, L.L., Ravi, N., Schneider, M., Scheller, M.F., Schneider, P., Mitre, M., Gouveia, M.D., Froemke, R.C., Chao, M.V., Young, W.S., et al. (2016). Oxytocin Enhances Social Recognition by Modulating Cortical Control of Early Olfactory Processing. Neuron 90, 609-621.

Roberts, S.A., Davidson, A.J., Beynon, R., and Hurst, J. (2014). Female attraction to male scent and associative learning: The house mouse as a mammalian model. Animal Behaviour 97, 313-321.

Roberts, S.A., Davidson, A.J., McLean, L., Beynon, R.J., and Hurst, J.L. (2012). Pheromonal induction of spatial learning in mice. Science 338, 1462-1465.

Schindelin, J., Arganda-Carreras, I., Frise, E., Kaynig, V., Longair, M., Pietzsch, T., Preibisch, S., Rueden, C., Saalfeld, S., Schmid, B., et al. (2012). Fiji: an open-source platform for biological-image analysis. Nat Methods 9, 676-682. 
Schneider, R.A., Costiloe, J.P., Howard, R.P., and Wolf, S. (1958). Olfactory perception thresholds in hypogonadal women: changes accompanying administration of androgen and estrogen. J Clin Endocrinol Metab 18, 379-390.

Sigl-Glockner, J., Maier, E., Takahashi, N., Sachdev, R., Larkum, M., and Brecht, M. (2019). Effects of Sexual Experience and Puberty on Mouse Genital Cortex revealed by Chronic Imaging. Curr Biol.

Stowers, L., and Logan, D.W. (2010). Olfactory mechanisms of stereotyped behavior: on the scent of specialized circuits. Curr Opin Neurobiol 20, 274-280.

Verhaeghe, J., Gheysen, R., and Enzlin, P. (2013). Pheromones and their effect on women's mood and sexuality. Facts Views Vis Obgyn 5, 189-195.

Welker, W.I. (1964). Analysis of Sniffing of the Albino Rat. Behaviour 22, 223-244.

Wojtowicz, J.M., and Kee, N. (2006). BrdU assay for neurogenesis in rodents. Nat Protoc 1 , 1399-1405.

Wysocki, C.J., Dorries, K.M., and Beauchamp, G.K. (1989). Ability to perceive androstenone can be acquired by ostensibly anosmic people. Proc Natl Acad Sci U S A 86, 79767978.

Ye, B., Huang, L., Gao, Z.L., Chen, P., Ni, H., Guan, S.D., Zhu, Y., and Wang, J.H. (2012). The Functional Upregulation of Piriform Cortex Is Associated with Cross-Modal Plasticity in Loss of Whisker Tactile Inputs. Plos One 7.

Young, L.J., Lim, M.M., Gingrich, B., and Insel, T.R. (2001). Cellular mechanisms of social attachment. Horm Behav 40, 133-138. 